\title{
Acoustic emission associated with the bursting of a gas bubble at the free surface of a non-newtonian fluid.
}

\author{
T. Divoux, V. Vidal†, F. Melo and J.-C. Géminard $\dagger$ \\ Departamento de Física, and Center for Advanced Interdisciplinary Research in Materials (CIMAT), \\ Universidad de Santiago de Chile (USACH), Av. Ecuador 3493, \\ Casilla 30\%, Correo 2, Santiago de Chile, Chile. \\ $\dagger$ Permanent address : Université de Lyon, Laboratoire de Physique, \\ Ecole Normale Supérieure de Lyon - CNRS, 46, Allée d'Italie, 69364 Lyon cedex 07, France.
}

\begin{abstract}
We report experimental measurements of the acoustic emission associated with the bursting of a gas bubble at the free surface of a non-newtonian fluid. On account of the viscoelastic properties of the fluid, the bubble is generally elongated. The associated frequency and duration of the acoustic signal are discussed with regard to the shape of the bubble and successfully accounted for by a simple linear model. The acoustic energy exhibits a high sensitivity to the dynamics of the thin film bursting, which demonstrates that, in practice, it is barely possible to deduce from the acoustic measurements the total amount of energy released by the event. Our experimental findings provide clues for the understanding of the signals from either volcanoes or foams, where one observes respectively, the bursting of giant bubbles at the free surface of lava and bubble bursting avalanches.
\end{abstract}

\section{INTRODUCTION}

A host of broad-interest phenomena involve bursting bubbles at fluid surfaces. In daily life, jam or purée cooking produces sonic bubbles that can project fragments at bursting. In the geophysical context, giant bubbles bursting at the top of a volcano vent, or at the surface of a lava lake, are examples whose understanding might be crucial for predicting volcanic activity [1, 2]. Although less considered, bubble bursting also occurs at the surface of aqueous foams [3] typically produced by wash- or beauty- products or even by poured beer. The analysis of acoustics emission is then a natural way of investigating bursting systems, revealing the collapse or bursting mechanism and properties of the fluid gas mixture [4, [5, 6, 7, [8, 9].

On the one hand, various statistical analysis of bursting noise have been carried out [6, 7, 10]. For instance, the sound pattern of collapsing foams was recently analyzed, revealing a log-normal distribution for the energy of events. For the events of highest acoustic energy, the distribution is however a power law suggesting that a "wide variety of bubble membranes areas is exploding" [7]. Consistently, the film rupture event seems to be independent of the bubble size and exhibits instead a correlation in space due to cascade bursting [9]. In turn, the typical frequency of the acoustic signal has been statistically correlated to the bubble size [7, 10]. The acoustic emission of a single standing spherical bubble has been recorded as well [10] and sophisticated high speed techniques have been used to elucidate the bursting dynamics of spherical smectic films [11]. However, detailed correlations of high speed images of foam bursting-films with the features of the acoustic emission have not yet been performed.

On the other hand, volcanologists have recorded the sound produced by astounding burstings, and have tried to infer, from the signal characteristics, the dynamical processes involved in these natural phenomena [12]. Previous laboratory experiments have intended to reproduce bubbles formation, rising and bursting in geometries presenting similarities with bubbles in magma conduit or lava lakes [13, 14]. Since the forces applied on lava have a time scale much larger than its relaxation time (about $4 \times 10^{-8} \mathrm{~s}$ for Strombolian magma [15]), most of model experiments have been performed in newtonian fluid 13, 14, 16]. However, departure from this newtonian behavior can occur if the magma contains crystals [16], which can be observed for example on Strombolian ejecta [17]. Some experiments, performed in more 'exotic' fluids like japanese curry or tomato sauce, have demonstrated the complex behavior of such fluids, and brought to the fore the non-trivial physical processes leading to sound generation - from Helmholz resonator type to bubble oscillation inside the fluid [18].

To understand the geometrical and dynamical aspects of sound produced by bursting bubbles having an elongated shape, we described recently the acoustic emission from an overpressurized cylindrical cavity, closed at one end by a fluid film [19]. We showed that the cavity geometry governs the frequency, the viscous dissipation and radiation are responsible for the wave damping and the acoustic energy depends not only on the energy initially loaded inside the cavity but also on the characteristic time associated with the film bursting.

Here, we present the analysis of the high-frequency acoustic-wave emitted by a bubble bursting at the free surface of a non-newtonian fluid. In such fluids, the complex rheology [20] is the source of puzzling phenomena including surface instabilities due to elastic effects [21], cusp at the tail of elongated rising bubbles [20, 22, 23, 24] and oscillations of falling spheres or rising air-bubbles [25, 26, 27]. We choose an experimental situation in which the bubble, generally elongated, exhibits a nearly- 

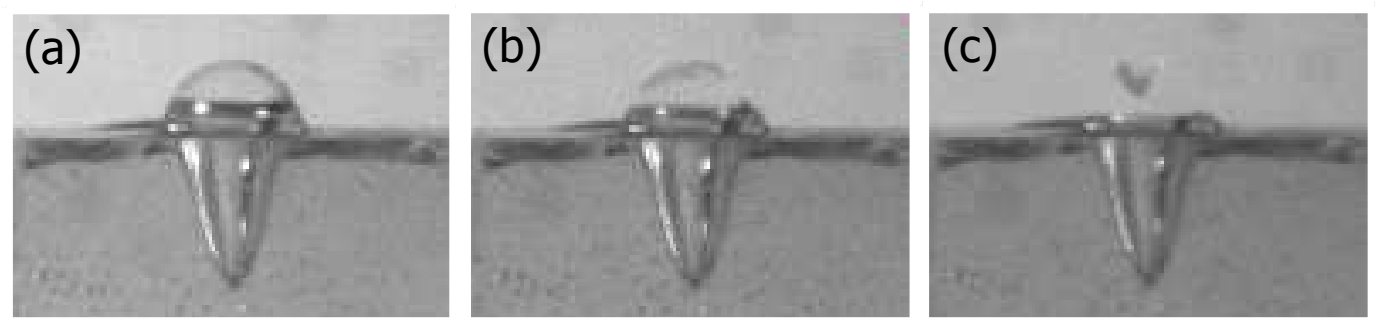

FIG. 1: Bursting event in the fast camera. (a) Initially, the bubble sits at the free surface. The film at the top is thinning due to the drainage. (b) The thin film suddenly breaks. One observes, in this specific example, that the film tears at its base. (c) As a result of the outgoing air flow and of the capillary forces, the remaining part of the bubble head is blown upwards and shrinks. The width of each image is $3 \mathrm{~cm}$ whereas the time-difference between them is $0.8 \mathrm{~ms}$. Here, the acoustic emission associated with the event does not last more than $2 \mathrm{~ms}$, during which the bubble body clearly does not significantly deform.

conical steady-shape during the rise toward the free surface. When the bubble reaches the free surface, a liquid film separates the bubble body from the surrounding air. This film thins, eventually breaks, and can display a complex behavior (see for instance [28, 29, 30, 31] for viscous films, and 32, 33] for soap films).

In the chosen experimental conditions, due to the nonnewtonian fluid properties, the bubble body does not significantly deform during the bursting event. We first link the characteristics of the radiated sound with bubble volume and shape, which is governed by the rheological properties of the fluid. Despite the conical shape of the bubbles, acoustic signals show a narrow frequency spectrum whose characteristic wavelength is linear in the bubble length, exhibiting a well-defined offset that is due to acoustic radiation [34]. Both the results are theoretically explained using linear acoustic [35, 36, 37]. Differences and similarities with the newtonian-fluid case are underlined. We then show that, if the rupture time of the viscoelastic film does not control the wave generation (i.e., it is fast enough), an optimal size for a 'sonic' bubble exists: The largest amplitude of the acoustic signal is recorded for a bubble having this optimal size.

We then show that, without a detailed knowledge of film-bursting dynamics, acoustic measurements are not a reliable method to access the total amount of energy released. Indeed, the amount of the energy transferred to the acoustic wave drastically depends on the characteristic time associated with the opening of the bubble [19], which is not controlled experimentally. This result might find interesting applications to aqueous foams as well, indicating that the statistic of energy released by bubble bursting-avalanches, recently characterized by acoustic emission [7, might be not only influenced by the distribution of the bubble sizes, but also by that of the rupture times. Consistently, our results suggest that bursting cascades might be triggered more likely by silent bubbles than by noisy ones. In this case, most of the potential energy loaded inside the cavity would contribute to larger distortions of bubble network.

\section{EXPERIMENTAL SETUP AND PROCEDURE}

The experimental setup consists of a vertical plexiglas container (square section $30 \times 30 \mathrm{~mm}$, height $88 \mathrm{~mm}$ ) filled with a transparent non-newtonian fluid up to the upper plane. Thanks to the transparent and planar walls of the container, the fluid can be imaged from the side without any optical distortion by means of a fast-camera (HiSIS 2002, KSV Instruments Ltd., up to $1220 \mathrm{im}-$ ages/sec, Fig. 10

In order to produce bubbles having a well-defined volume $V$ (from 0.1 to $1.5 \mathrm{~mL}$ ), a chosen amount of air is rapidly injected by means of a syringe pump connected to the container by a hole drilled at the center of the lower plane. After injection, the bubble rises in the fluid, reaches the upper free surface (Fig. 1 \& 21) and finally bursts, producing a characteristic audible sound.

To characterize this phenomenon, we record the acoustic emission by means of a microphone (ATM33a, Audio Technica associated with a preamplifier, Eurorack UB802) which is located $3 \mathrm{~cm}$ away from the gel freesurface, with a 45-degrees inclination from the vertical. The position of the microphone shall remain identical for all the experimental results reported herein.

The chosen non-newtonian fluid is obtained by diluting a commercial hair-dressing gel (Gel fijador de cabello, for men, Camel White ${ }^{\circledR}$ ) in pure water. This latter choice is mainly justified by the fact that, in such a fluid, the air bubbles usually exhibit a nice vertically-elongated shape, terminated by a cusp at the bottom, which significantly differs from the rounded shape usually observed in a newtonian fluid [20]. In addition, one can easily be supplied with large quantities of fluid, reproducible mixtures are rather easy to prepare and they are stable in the time. The non-newtonian character of the fluid is more or less pronounced depending on the concentration, $c$, of gel in the mixture (from 25 to $40 \%$ in volume). All the solutions are obtained after the mixing of the two components during half a day by means of a magnetic stirrer. Afterwards, the small bubbles that still remain trapped in the fluid 
are eliminated by placing the solution in an ultrasonic bath for several hours. In order to avoid any memory effect, the gel is stirred and let at rest for a few minutes between two bubble rises. The mixtures are likely to be subjected to drying: In order to avoid any significant change in the overall concentration $c$, each sample is used only for 4 days.

\section{PRELIMINARY OBSERVATIONS : BUBBLE SHAPE AND BURSTING DYNAMICS}

Before analyzing in details the acoustic emission associated with the bursting of a single bubble at the free surface, one must first pay attention to the bubble shape and dynamics.

First, depending on the concentration $c$ and on the volume $V$, one observes two qualitative different steady shapes of the bubbles which rise up in the bulk of the fluid. Indeed, for low gel concentration (typically $c<30 \%$ ), the bubbles exhibit an almost spherical shape, similar to that observed in newtonian fluids. On the contrary, for larger concentration (typically $c>30 \%$ ), the bubbles exhibit an elongated shape with a cusped tail, as already observed in non-newtonian fluids [20, 22, 23, 24]. During the rise, one also notices oscillations in the bubble shape, as previously pointed out in the literature [27].

The shape of the bubble which bursts at the free surface qualitatively exhibits almost the same transition. For small concentration $c$, one observes a rounded bubble on the last image previous to the bursting (Fig. 2a) whereas, for a larger concentration $c$, a cusp is clearly observed (Fig. 2d). However, we point out that at intermediate concentration (typically $c \sim 30 \%$ ) the shape of the bubble, when bursting, also depends on its dynamical behavior when reaching the free surface and, thus, on its volume $V$ : For small volume $(V<0.25 \mathrm{~mL})$, only rounded bubbles are observed whereas, for large volume $(V>0.40 \mathrm{~mL})$, the bubbles always exhibit a cusp; In the intermediate range $(0.25 \leq V<0.40 \mathrm{~mL})$, both types of bubble can be observed for the same volume $V$. Qualitatively, the capillary forces are large enough to maintain the small bubbles in equilibrium at the free surface so that a bubble, which initially exhibits a cusp while rising, deforms and equilibrates before the thin film that encloses the inside air breaks due to the drainage. No cusp is then observed. On the contrary, the capillary forces are not large enough to maintain the largest bubbles in equilibrium at the free surface. As a consequence, the tail of the bubble does not significantly deform at the free surface before the thin film at the top breaks, due to the increase of its surface area and not to the drainage, in this case. A cusp is thus systematically observed. In the intermediate case, the capillary forces are likely to maintain the bubble at the free surface but, because of some premature ruptures of the thin film at the top, one can observe a bubble exhibiting either a rounded bottom or a cusp.

The equilibrium shape or the dynamical behavior of the bubble at the free surface, which would deserve an extensive study to be accounted for, are not the aim of the present work. From the qualitative description of the bubble behavior at the free surface presented above, we shall only remember that the bubble are elongated and exhibit a cusp in most of the experimental conditions and that, depending on the volume, the thin film at the top might break either because of the drainage or of the increase in its surface area. In what follows, we shall only analyze the acoustic signal associated with the bursting event with regard to the shape and dynamics of the bubble at the free surface.

\section{THE ACOUSTIC SIGNAL}

The bursting event is systematically associated with the emission of a sound wave, characterized by a welldefined frequency (Fig. 2).

Qualitatively, the sudden bursting of the thin film at the top excites a resonant pressure-wave in the bubble body which is initially overpressurized. The phenomenon is similar to that thoroughly described in Ref. [19], where the acoustic emission associated with the bursting of a thin soap film that initially closes a cylindrical overpressurized cavity is analyzed in details. In our experimental case, due to the viscoelastic properties of the fluid, the bubble body is generally elongated and the bubble wall does not significantly deform during the characteristic duration of the sound emission (Fig. 11). The opened bubble-body thus selects resonant modes among which the fundamental is the most intense. Due to the radiation at the open end, one records outside the cavity a sound wave exhibiting well-defined frequency and duration which are the subject of the analysis presented below.

\section{Acoustic wavelength}

In figure 3, we report the wavelength, $\lambda$, associated with the acoustic wave in air as a function of the bubble length, $L$, as defined in figure 4. Taking the whole set of the experimental data obtained for different gel concentration $c$ and bubble volume $V$ into account, one observes experimentally that $\lambda$ increases almost linearly with $L$ according to $\lambda \simeq \lambda_{0}+(2.8 \pm 0.1) L$ with $\lambda_{0} \simeq(1.6 \pm 0.1) \mathrm{cm}$. The experimental slope $d \lambda / d L \simeq 2.8$ deserves to be contrasted with the slope $d \lambda / d L=4$ obtained in the case of a cylindrical cavity [19, 36]. In the same way, a straightforward analysis of the acoustic problem leads to $d \lambda / d L=2$ in the case of a conical cavity. In order to account for the experimental slope, let us now consider the acoustic 
(a)
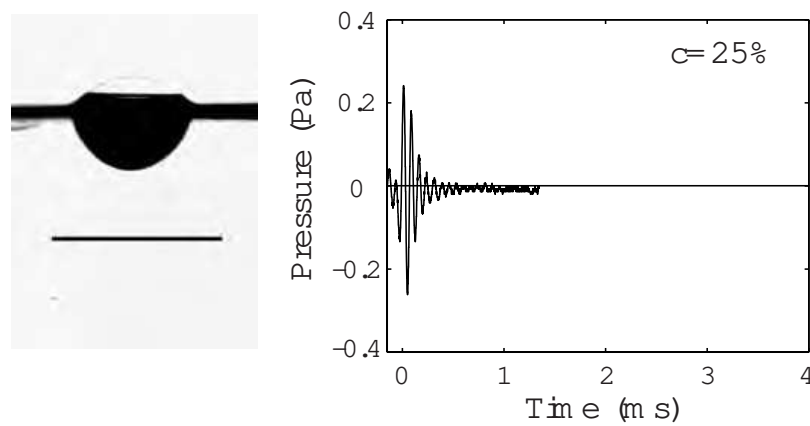

(c)

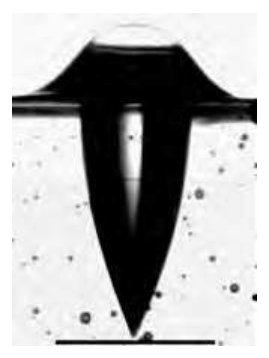

(b)
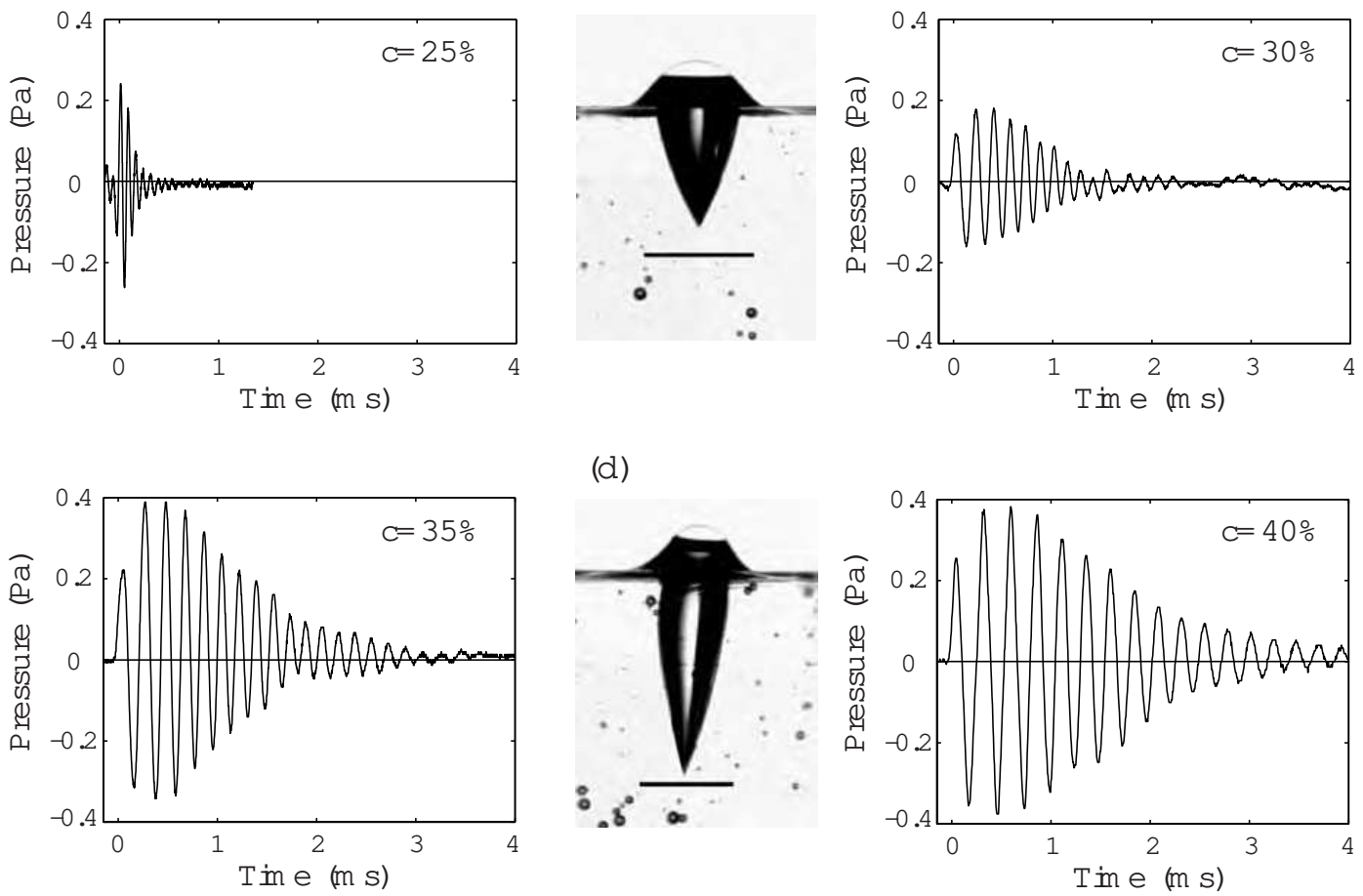

(d)

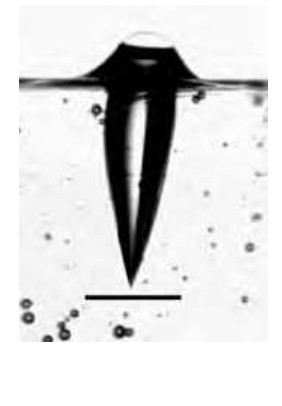

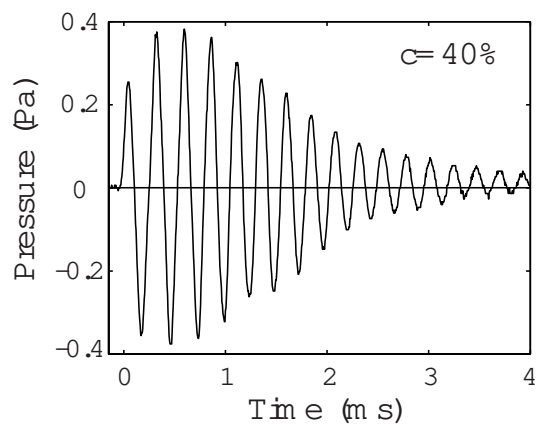

FIG. 2: Images of the bubble right before bursting and associated acoustic signals. In the images (scale bar: $1 \mathrm{~cm}$ ), one notices that the bubbles are more elongated when the gel concentration $c$ is larger. In addition, we report the signal from the microphone. We observe that the typical frequency decreases and the characteristic duration of the acoustic emission increases when the bubble length is increased.

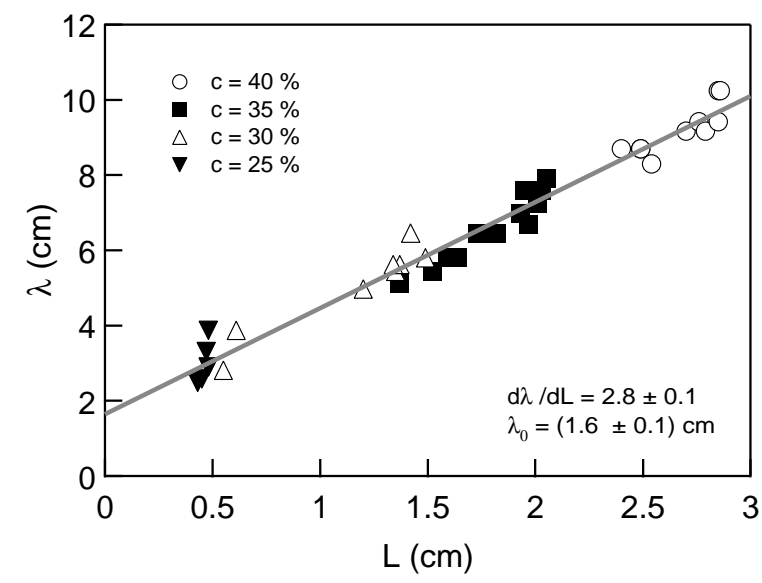

FIG. 3: Wavelength $\lambda$ vs. bubble length $L$. The resonant wavelength increases linearly with the bubble length, estimated from the open aperture to the tail. The offset $\lambda_{0}$ is accounted for by the radiation at the open-end.

wave inside the bubble, taking into account the cusp at the tail and the overall shape of the cavity.

Because of the cusp at the tail, the bubble body resem- bles a cone. As a consequence, we shall work in a system of spherical coordinates centered in $O$, at the cusp. Let $M$ be a point of the bubble wall. The shape of the bubble, assumed to be axisymmetric, can be accounted for by the angle $\alpha(r)$ between the vector $\overrightarrow{O M}$ and the symmetry axis $(r \equiv O M$, Fig. 任). For instance, a conical bubble would be described by $\alpha(r)=\alpha_{0}$, a constant. In a first approximation, assuming that the variation of the bubble cross-section does not depend too rapidly (in comparison to the wavelength) on the radius $r$, we can write the equation for the pressure field $P(r, t)$, assumed to depend only on the distance $r$,

$$
\begin{aligned}
\frac{1}{v^{2}} \frac{\partial^{2} P(r, t)}{\partial t^{2}} & =\frac{1}{r^{2}} \frac{\partial}{\partial r}\left(r^{2} \frac{\partial P(r, t)}{\partial r}\right) \\
& +\frac{\partial P(r, t)}{\partial r} \frac{d}{d r}(\log [1-\cos \alpha(r)])
\end{aligned}
$$

where $v$ stands for the velocity of the sound in air. The equation (11), written in spherical coordinates, governs the propagation of a pressure wave in an acoustic horn [36, 37] whose profile is described by the function $\alpha(r)$. First, a velocity node locates at $O$. Second, neglecting the radiation at the open end, we can, in a first approximation, assume that a pressure node locates in the plane of the aperture $[P(L)=0$, note here that the condi- 
tion of zero pressure in the output plane is not compatible with the geometry of the pressure field. However, in a first approximation, to within a term of the order of $\Phi / L$, where $\Phi$ stands for the aperture diameter, this assumption provides a good estimate of the resonant wavelength.] For instance, in the case of a conical cavity $\left(\alpha=\alpha_{0}\right)$, the solution at the frequency $\omega$ is $P(r, t)=\frac{\sin k r}{k r} e^{j \omega t}$ with $k L=\pi$, which leads to $\lambda=2 L$ for the fundamental $\left(k \equiv \frac{2 \pi}{\lambda}\right)$. Experimentally, the bubble is not conical and its profile is successfully interpolated by the phenomenological function $\alpha(r)=\alpha_{0} \cos \left(\beta \frac{r}{L}\right)$, where $\alpha_{0}$ denotes half the angle at the bubble tail (Fig. 4a). In this case, the equation (11) must be solved numerically. The coefficient $\beta$ accounts for the diameter $\Phi$ of the aperture at the top surface, according to $\alpha_{0} \cos \left(\beta \sqrt{1+\left(\frac{\Phi}{2 L}\right)^{2}}\right)=\arctan \left(\frac{\Phi}{2 L}\right)$. In Fig. $4 \mathrm{~b}$, we report the slope $\frac{d \lambda}{d L}$ as a function of $\alpha_{0}$ for different values of the ratio $\Phi / L$. For instance, one obtains $\frac{d \lambda}{d L}=2.8$ for $\alpha_{0}=\frac{\pi}{4}$ and $\frac{\Phi}{L}=\frac{1}{2}$, and $\frac{d \lambda}{d L}=2.9$ for $\alpha_{0}=\frac{\pi}{8}$ and $\frac{\Phi}{L}=\frac{1}{4}$, which corresponds to the typical values of these two parameters in our experimental conditions. Thus, in spite of the slight dependance of the slope $\frac{d \lambda}{d L}$ on the $\alpha_{0}$ and $\Phi / L$, the experimental wavelength $\lambda$ is observed to depend almost linearly on the bubble length $L$ with $\frac{d \lambda}{d L} \simeq 2.8$ for the whole set of experimental data reported in Fig. 3 .
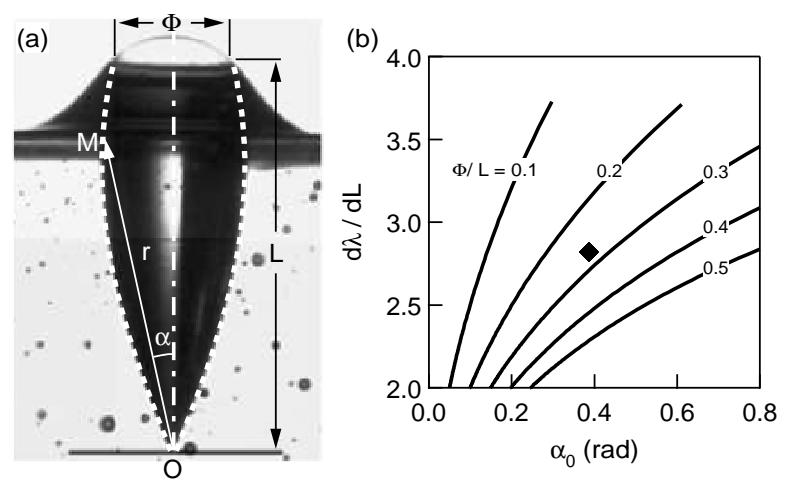

FIG. 4: (a) Image of a bubble and definitions. The dotted curve corresponds to the proposed interpolation of the bubble profile by $\alpha(r)=\alpha_{0} \cos \left(\beta \frac{r}{L}\right)$ with $\alpha_{0}=0.378$ and $\beta=1.11$. (b) Slope $\frac{d \lambda}{d L}$ vs. $\alpha_{0}$ for different ratio $\frac{\Phi}{L}$. The slope $\frac{d \lambda}{d L}$ is obtained from the solution of Eq. 1 taking into account the profile of the bubble wall. The full diamond corresponds to the bubble in (a).

However, the simple acoustic model presented above fails in accounting for the finite offset $\lambda_{0} \simeq 1.6 \mathrm{~cm}$ clearly observed in figure 3. As already pointed out, we arbitrarily assumed that a pressure node locates in the plane of the open end of the bubble, this latter condition being incompatible with the geometry of the pressure field inside the bubble. In order to recover the value $\lambda_{0}$ of the off- set, one must consider the diffraction of the sound wave by the aperture. Indeed, the boundary condition in the output plane is imposed by the continuity of the pressure and velocity fields in this very plane, and one must also consider the acoustic wave outside the cavity. The structure of the acoustic wave resulting from the diffraction of a planar wave by a circular aperture (diameter $\Phi$ ) has been determined by several authors. As a result, to the first order in $\Phi / \lambda$, one obtains the acoustic impedance of the outer acoustic wave $\mathcal{Z}_{\text {out }} \simeq \zeta j k \Phi$, where $\zeta=\frac{4}{3 \pi}$ for of a flanged aperture [37] and $\zeta=0.3$ for an unflanged aperture [34]. In the case of the diffraction of a spherical wave, we expect these results to hold true [the correction due to the curvature of the pressure field is expected to be of the order of $\left(\frac{\Phi}{L}\right)^{2}$.] In addition, we can estimate the acoustic impedance of the inner acoustic wave, $\mathcal{Z}_{\text {in }}$, by calculating the average pressure and velocity in the aperture plane from the solution of equation (11), the boundary condition at $O$ being taken into account. Then, insuring the continuity of the pressure and velocity fields in the aperture plane, one can determine the resonant wavelength $\lambda$. In the case of a conical cavity, writing $\mathcal{Z}_{\text {out }} /(\rho v) \simeq \zeta j k \Phi$, one gets $\lambda=2 L+2 \pi \zeta \Phi$. Thus, in the case of a conical cavity and of a flanged aperture, we estimate $\lambda_{0} \simeq \frac{8}{3} \Phi$ (We remind here that one would expect $\lambda_{0} \simeq \frac{4}{3 \pi} \Phi$ for a cylindrical cavity [19].) In the case of the bubble, the impedance $\mathcal{Z}_{\text {in }}$ must be evaluated numerically as the equation (11) does not exhibit any simple analytical solution. Here, we only aim at elucidating the physical origin of the offset $\lambda_{0}$. As a consequence, we only point out that, if due to the radiation at the open end, we expect $\lambda_{0}=(1.5 \pm 0.5) \mathrm{cm}$ for the experimental range of the aperture diameter $(\Phi \in[0.4,0.7] \mathrm{cm})$. From the agreement of this last estimate with the experimental value of $\lambda_{0}$, we conclude that the offset originates from the radiation of the resonant acoustic wave at the open end.

\section{Damping of the acoustic signal}

In order to account for the damping of the acoustic wave, we report in Fig. [5 the typical duration, in number of periods, $n$, of the acoustic signals. This choice is explained by the difficulty in defining precisely the signal duration because of the rather complex envelop of the acoustic signal. For an exponential decay over the characteristic time $\tau$, we would expect $n=n_{0}+\frac{\omega \tau}{2 \pi}$, so that the experimental value of $n$ provides an estimate of the characteristic time $\tau$ to within an offset. The damping of the acoustic signal might be governed by several physical processes, among them the partial reflection at the cusp, the viscous dissipation at the side walls and the diffraction at the open end. Each of the processes would lead to a different dependence of the duration on the geometry of the bubble. The partial reflection at the cusp would 
lead to constant $\omega \tau$ whereas the viscous dissipation would lead to $\omega \tau \propto \Phi \sqrt{\omega}[19]$ and, thus, to a decrease in $n$ for increasing $L$. On the contrary, one observes experimentally an increase in $n$ with the bubble length $L$. In order to account for the radiation, we can further expand the acoustic impedance $\mathcal{Z}_{\text {out }}$ to the second order in $k \Phi$ and write $\mathcal{Z}_{\text {out }} /(\rho v) \simeq \zeta j k \Phi+\xi(k \Phi)^{2}$ where $\xi$ is a constant. For the radiation of a planar wave at a flanged (resp. unflanged) aperture, $\xi=\frac{1}{8}$ (resp. $\frac{1}{16}$ ) [34, 37]. As already mentioned, we expect the curvature of the wavefront to slightly alter the value of the impedance to within a term of the order of $(\phi / L)^{2}$ but we do expect $\xi$ to remain of the same order. The solution of the equation 1, taking into account the boundary condition at the open end, then leads to $\omega \tau=\frac{1}{\xi} \frac{L^{2}}{\pi \Phi^{2}}$ and thus to $n=n_{0}+\frac{1}{\xi} \frac{L^{2}}{2 \pi^{2} \Phi^{2}}$. Our measurements are not accurate enough to make it possible to determine the experimental slope $\frac{1}{\xi}$ but we point out that they are compatible with the value $\xi=\frac{1}{8}$ (Fig. 5). We can thus conclude that the damping of the acoustic signal is mainly governed by the radiation at the open end of the bubble.

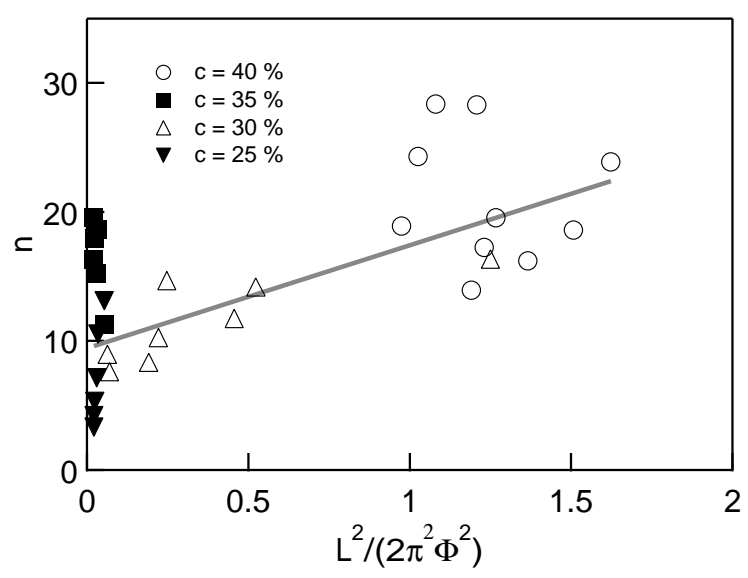

FIG. 5: Characteristic duration of the sound emission $n$, in number of periods, vs. $L^{2} / \Phi^{2}$. The dependence of $n$ on the ratio $L / \Phi$ demonstrates that the damping of the acoustic signal is mainly governed by the radiation at the open end (The slope of the grey line is 8 , thus corresponding to $\xi=\frac{1}{8}$, see text.)

\section{Acoustic energy}

At this point, it is particularly interesting to focus on the energy associated with the acoustic emission. From the pressure signal, $P(t)$, provided by the microphone, one can estimate the total amount of energy released in the acoustic signal at the fundamental frequency, $E_{a}$. Assuming that the acoustic wave outside the bubble is almost spherical, centered at the bubble aperture, in the half-space above the free surface, we can write:

$$
E_{a} \simeq \frac{2 \pi d^{2}}{\rho v} \int_{t=0}^{\infty} P(t)^{2} d t
$$

where, we remind, $d$ stands for the distance from the microphone to the bubble aperture. As $E_{a}$ is expected to depend on the volume $V$ and of the initial overpressure $\delta P$ of the air inside the bubble before the bursting, let us now consider an estimate of the total amount of released energy, $E_{T}$, assuming a rapid expansion of air:

$$
E_{T}=\frac{1}{2} \frac{V \delta P^{2}}{\rho v^{2}}
$$

Experimentally, the volume $V$ is obtained from the injected volume of air but the overpressure $\delta P$ is rather difficult to estimate. However, we measured the surface tension $\gamma=(25 \pm 5) \mathrm{mN} / \mathrm{m}$ of the gel-water interface for all concentration $c$ and we can estimate the tension of the thin film at the top, previous to the bursting event, to be about $2 \gamma$. Measuring the radius of curvature of the bubble head, $\mathcal{R}$, from the image, we can estimate $\delta P \simeq 4 \gamma / \mathcal{R}$. We point out that the corresponding value of $E_{T}$ must be considered with caution (Laplace's law might not be valid for bubbles bursting without reaching equilibrium at the free surface). However, it makes possible to estimate - and therefore, to further discuss - the energy of the acoustic emission.

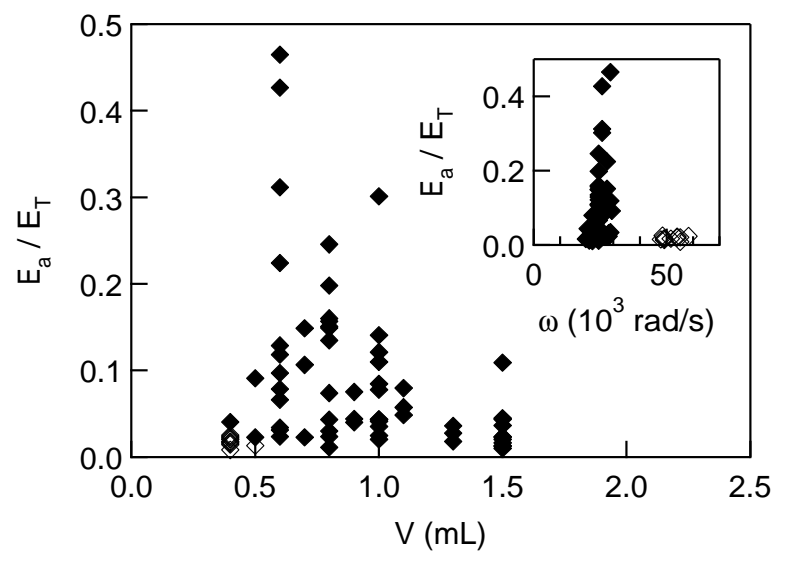

FIG. 6: Ratio $E_{a} / E_{T}$ vs. volume $V$. At a given volume $V$, the acoustic energy $E_{a}$ does not account for the amount of energy released by the bursting bubble. Inset: Ratio $E_{a} / E_{T}$ vs. frequency $\omega$ : The experimental results clearly demonstrate that the bubbles can be separated in two categories: small bubbles that sit at the free surface before bursting (open symbols) and larger ones that cross the interface without stopping (full symbols).

We report first $E_{a} / E_{T}$ as a function of the bubble volume $V$ (Fig. 6). One clearly observes that the data points are widely distributed, which indicates that the acoustic 
energy $E_{a}$ does not image the total amount of energy released by the bursting event, $E_{T}$. However, the envelop of the data points indicates that, at intermediate volume, in some cases, a large part of $E_{T}$ is transferred to the acoustic mode, which explains why some of the bubbles are so loud whereas others are barely audible. As already discussed in Ref. [19], the transfer of the energy intially loaded in the bubble to the acoustic modes is mainly governed by the characteristic time associated with the rupture of the bubble head: The opening of the cavity is efficient in exciting the inner resonant modes only if rapid enough. For the smallest volumes, the bubble rises and stops at the free surface. The film that closes the cavity bursts after drainage. Even small, the characteristic time associated with the cavity opening is long compared to the acoustic period because the bubble body is very short. As a consequence, $E_{a} / E_{T}$ is relatively small. In the opposite limit, the bubbles are large and cross the interface without stopping. The bursting results from the breaking of the bubble head which is torn apart due the bubble dynamics. In this case, the film is not as thin as one would obtain as the result of the drainage and the characteristic opening-time is long compared to the period associated with the resonant modes, even if the bubble body is long. Again, the ratio $E_{a} / E_{T}$ is relatively small. The optimal conditions are reached when the volume of the bubble is such that the bubble crosses the interface slowly enough for the film to have time to thin but rapidly enough for the bubble tail not to disappear (this leads to a significant increase of the resonant frequency). This latter conclusion is supported by the data reported in Fig. 6, inset. One observes, reporting $E_{a} / E_{T}$ as a function of the resonant frequency $\omega$, that the data can be separated in two groups: bubbles that sit at the interface before bursting and bubbles that cross the interface without stopping. We clearly note that $E_{a} / E_{T}$ is maximum for bubbles crossing dynamically the free surface.

From these remarks on the acoustic energy, we would like to point out that the acoustic energy in the fundamental mode drastically depends on the dynamics of the thin film rupture and that, as a consequence, measuring the acoustic energy is not enough for obtaining a good estimate of the total energy release.

\section{CONCLUSION}

Motivated both by the will to understand the physical processes involved when a bubble bursts at the surface of a non-newtonian fluid, and the hope to use the acoustics as a tool to investigate natural systems such as volcanoes the relationship between the acoustic wave and the rheological properties of lava, we have investigated the bursting of bubbles at the free surface of a gel solution, diluted at different concentration.
We have shown that, at large-enough gelconcentration, the bubble, which is elongated, acts as a motionless resonator and, thus, exhibits a welldefined acoustic-frequency at bursting. The amplitude of the acoustic wave emitted at bursting depends on various parameters: gel concentration, volume of the bubble, and film rupture time. In spite of the observed clear transition between a static regime, where the small-volume bubble remains trapped at the surface, and a dynamical regime, where the high bubble-rising velocity makes the bubble go through the surface and burst directly, the problem remains rich and complex, in particular, due to the unpredictable film rupture time, which directly influences the amplitude and energy of the acoustic signal.

From a practical point of view, we can raise the following question: What pieces of information can we infer from acoustic measurements, if they are the only available data? From our study, we can conclude that the frequency of the signal gives a direct access to the bubble length. However, any attempt to interpret the amplitude and energy of the acoustic signal would surely lead to strong misinterpretation. Indeed, we have shown that the same experimental conditions (gel concentration, bubble volume) can lead to completely different acoustic-signals at bursting, due to the high sensitivity to the film bursting dynamics.

\section{ACKNOWLEDGEMENTS}

The collaborative research was supported by CNRS/CONICYT project \#18640 and Conicyt-Chile FONDAP project \#11980002.

[1] E. A. Parfitt, J. Volcanol. Geotherm. Res. 134, 77 (2004).

[2] H. M. Gonnermann and M. Manga, Annu. Rev. Fluid Mech. 39, 321 (2007).

[3] D. Weaire and S. Hutzler, The Physics of Foams (Oxford University Press, New York, 1999).

[4] A. Prosperetti and H. N. Oguz, Annu. Rev. Fluid Mech. 25, 577 (1993).

[5] T. G. Leighton, The Acoustic Bubble (Academic Press, San Diego, 1994).

[6] W. Müller and J.-M. di Meglio, J. Phys.: Condens. Matter 11, L209 (1999).

[7] N. Vandewalle, J. F. Lentz, S. Dorbolo, and F. Brisbois, Phys. Rev. Lett. 86, 179 (2001).

[8] N. Vandewalle and J. F. Lentz, Phys. Rev. E 64, 021507 (2001).

[9] N. Vandewalle, H. Caps, and S. Dorbolo, Physica A 314, 320 (2002).

[10] J. Ding, F. W. Tsaur, A. Lips, and A. Akay, Phys. Rev. E. 75, 041601 (2007).

[11] F. Müller, U. Kornek, and R. Stannarius, Phys. Rev. E. 75, 065302(R) (2007). 
[12] J. B. Johnson, R. C. Aster, M. C. Ruiz, S. D. Malone, P. J. McChesney, J. M. Lees and P. R. Kyle, J. Volcanol. Geotherm. Res. 121, 115 (2003).

[13] C. Jaupart and S. Vergniolle, Nature 331, 58 (1988); C. Jaupart and S. Vergniolle, J. Fluid Mech. 203, 347 (1989).

[14] M. Ripepe, S. Ciliberto and M. Della Schiava, J. Geophys. Res. 106, 8713 (2001).

[15] S. L. Webb and D. B. Dingwell, J. Geophys. Res. 95, 15695 (1990).

[16] S. Vergniolle and G. Brandeis, J. Geophys. Res. 101, 20433 (1996).

[17] L. Francalanci, P. Manetti and A. Pecerillo, Bull. Volcanol. 51, 335 (1989).

[18] M. Ichihara, T. Yanagisawa., Y. Yamagishi, H. Ichikawa, and K. Kurita, Japan Earth and Planetary Science Joint Meeting, Abstract (CD-ROM) A111-P004 (2005).

[19] V. Vidal, J.-C. Géminard, T. Divoux and F. Melo, Eur. Phys. J. B 54, 321 (2006).

[20] R. B. Bird, R. C. Armstrong and O. Hassager, Dynamics of Polymeric Liquids, Vol. I and II (Wiley, New York, 1987).

[21] T. Podgorski and A. Belmonte, J. Fluid Mech. 460, 337 (2002).

[22] O. Hassager, Nature 279, 402 (1979).

[23] Y. Liu, T. Liao and D. D. Joseph, J. Fluid Mech. 304, 321 (1995).

[24] R. P. Chhabra, Bubbles, drops, and Particles in Non Newtonian fluids, 2nd edition (Chemical industries series
\#113, Taylor \& Francis, 2007).

[25] N. Z. Handzy and A. Belmonte, Phys. Rev. Lett. 92, 124501 (2004).

[26] A. Jayaraman and A. Belmonte, Phys. Rev. E 67, 065301 (2003).

[27] A. Belmonte, Rheol. Acta 39, 554 (2000).

[28] K. Dalkoni-Veress, B. G. Nickel, C. Roth et al., Phys. Rev. E 59, 2153 (1999).

[29] G. Debrégeas, P. Martin and F. Brochard-Wyart, Phys. Rev. Lett. 75, 3886 (1995).

[30] G. Debrégeas, P.-G. de Gennes and F. Brochard-Wyart, Science 279, 1704 (1998).

[31] J. R. Dutcher, K. Dalkoni-Veress, B. G. Nickel et al., Macromol. Symp. 159, 143 (2000).

[32] P. Warszyński, B. Jachimska and K. Malysa, Colloids Surfaces A: Physicochem. Eng. Aspects 108, 321 (1996); B. Jachimska, P. Warszyński and K. Malysa, Colloids Surfaces A: Physicochem. Eng. Aspects 143, 429 (1998).

[33] J. K. Angarska and E. D. Manev, Colloids Surfaces A: Physicochem. Eng. Aspects 190, 117 (2001).

[34] H. Levine and J. Schwinger, Phys. Rev. 73, 383 (1948).

[35] L. D. Landau and E. M. Lifshitz, Course in Theoretical Physics: Fluid Mechanics, vol.6, 536 pp. (Pergamon, Tarrytown, N.Y., 1987).

[36] A. Pierce, Acoustics - An introduction to its physical principles and applications (ASA, New-York, 1989).

[37] Lord Rayleigh, Theory of Sound (Macmillan, 1940). 